\section{The Side Effects of Metformin - A Review}

\author{
Shraboni Ghosal ${ }^{1 *}$ and Shomik Ghosal ${ }^{2}$ \\ ${ }^{1}$ Nutrition Consultant, Newcastle under Lyme, Staffordshire, UK \\ ${ }^{2}$ Paediatric Consultant, University Hospital of North Midlands, Staffordshire, \\ UK
}

\begin{abstract}
Type 2 Diabetes mellitus (T2DM) needs to be managed with medications along with a healthy lifestyle to stabilize levels of blood glucose. Metformin is an oral biguanide and a first choice anti-hyperglycaemic medication for T2DM. It reduces levels of blood glucose by inhibition of glucose production in the liver. Metformin helps the body to restore its ability to respond to insulin and is generally a safe and effective treatment. Other than glycaemic control, metformin also improves insulin resistance, dysfunction of the endothelium, oxidative stress, and haemostasis and lipid profiles.

Metformin can help to avoid complications as a result of high blood glucose, such as diabetic nephropathy, neuropathy and retinopathy. Metformin can adversely affect renal functions of T2DM patients and chronic kidney disease can develop. The serious side effect of lactic acidosis is rare but can develop because metformin can accumulate. This review discusses the possible side effects of metformin, however patients taking metformin may or may not experience any or all of them. Metformin should be used carefully in the elderly and in patients who have trauma, fever, surgery, heart failure, impaired kidney or liver functions. Treatment with metformin should be stopped before any type of surgery.
\end{abstract}

Keywords: Gastrointestinal disorders; Lactic Acidosis; Metformin; Side effects; Type 2 Diabetes

\section{Introduction}

Diabetes mellitus has associations with different metabolic conditions and its growing prevalence worldwide is a cause for concern. In 2016, diabetes caused 1.6 million deaths and in 2014, diabetes

*Corresponding author: Shraboni Ghosal, Nutrition Consultant, Newcastle under Lyme, Staffordshire, UK, E-mail: castlediet1@gmail.com

Citation: Ghosal S, Ghosal S (2019) The Side Effects of Metformin - A Review. J Diabetes Metab Disord 6: 030.

Received: September 12, 2019, 2019; Accepted: October 16, 2019; Published: October 23, 2019

Copyright: ( $) 2019$ Ghosal S and Ghosal S. This is an open-access article distributed under the terms of the Creative Commons Attribution License, which permits unrestricted use, distribution, and reproduction in any medium, provided the original author and source are credited. developed in approximately $8.5 \%$ of people who were 18 years or above [1]. High blood glucose caused another 2.2 million deaths in 2012 [1]. It is an economic burden worldwide because it causes a broad range of complications and is a main cause of mortality as well as morbidity. Diseases such as neuropathy, nephropathy and retinopathy result from its long-term complications [2]. The hormone insulin is produced in the pancreas and regulates blood glucose. The majority ( $90 \%$ of people) who have diabetes have Type 2 Diabetes mellitus (T2DM) [3]. T2DM is caused due to a fault in the action or secretion of insulin or both combined and is chronic and gradual [4].

In T2DM, not enough insulin is produced by the pancreas or the insulin produced cannot be used efficiently [1]. This causes elevated plasma glucose concentrations or hyperglycemia. The development of hyperglycaemia is preceded by years of Insulin Resistance (IR) and hyperinsulinemia. Some of the symptoms of hyperglycemia include polyuria, polydipsia, quick breathing, fruity breath odor, confusion and drowsiness. Hyperglycaemia can seriously damage the nerves, blood vessels and many of the body systems over time [1]. Therefore, maintaining glycaemic control is essential in treating T2DM [5]. Some people with T2DM can achieve their target blood sugar levels with lifestyle interventions alone however others may need medication to help manage their levels of blood glucose.

\section{What is Metformin and how does it Work}

The most common medication used for treating T2DM is metformin (dimethylbiguanide), an oral antihyperglycaemic agent [6]. It is the first line pharmacological treatment for T2DM [7]. Approximately 380 million patients have diabetes worldwide, out of which metformin is used by about 120 million [8,9]. Metformin is derived from guanidine found in Galega officinalis [10] and for over forty years, it has been in clinical use [4]. Metformin received approval in 1994 by the US Food and Drug Administration (FDA), as a drug that is prescribed for treating diabetes. The American and European guidelines recommend metformin therapy in T2DM, in addition to lifestyle interventions [11]. The safety profile of metformin is good and it is recommended because of its low cost and associated safety [12].

Metformin is a biguanide and inhibits glucose production in the liver, thus lowering levels of blood glucose [11]. In adults with T2DM, metformin is used either alone or along with insulin or other antidiabetic drugs and lifestyle modifications. A systematic review showed that metformin lowers levels of blood glucose after food consumption and also lowers Fasting Plasma Glucose (FPG) in patients who have T2DM [13]. Metformin gets absorbed quickly from the small intestine and the highest levels of plasma concentration are observed in about two hours [14,15]. The administration of metformin 30 mins before a meal was found to work the best in decreasing postprandial hyperglycemia [16]. Metformin improves carbohydrate and lipid metabolism and decreases glycaemic parameters considerably [17]. A meta-analysis found that metformin reduces FPG levels by $2.8 \mathrm{mmol} / \mathrm{L}-3.9 \mathrm{mmol} / \mathrm{L}(50 \mathrm{mg} / \mathrm{dL}-70 \mathrm{mg} / \mathrm{dL})$ and glycated haemoglobin (HbA1c) levels by about $1.5 \%-2.0 \%$ [15]. It suppresses 
gluconeogenesis, inhibits glycogenolysis and stimulates glycolysis [18]. Metformin also reduces serum free fatty acid concentrations [19], improves IR and corrects dyslipidemia.

Metformin may have protective effects on pancreatic islet cells [20]. The Diabetes Prevention Program (DPP) [21] found that over a period of 3 years, metformin decreased the progression of diabetes by $31 \%$ in adults who had Impaired Glucose Tolerance (IGT) and also improved the functioning of the beta cells [21,22]. However, metformin works only when endogenous insulin is present and therefore acts when some functional pancreatic islet cells are still remaining [23]. Insulin secretion is not stimulated directly by metformin [24], however insulin sensitivity is improved by metformin because it enhances uptake of glucose in the peripheral tissues and glucose usage in the muscle tissues $[23,25]$. As a result, the amount of lactate in the enterocytes increases [26]. Metformin's actions have been found to be less on dipeptidyl peptidase-4, but the levels of plasma glucagon-like peptide-1 (GLP-1) are enhanced by it [26].

\section{Formulation \& Dosage of Metformin}

Metformin (generic name) is available in different formulations such as, tablets, capsules, oral suspensions, oral solutions or modified-release tablets [23]. Metformin's availability under different brand names include diagemet, bolamyn, glucophage, metabet, glucient [27], glucophageXR, fortamet and glumetza [28]. Riomet is the brand name for liquid metformin [27]. The immediate-release tablet is available as the brand-name of glucophage. Metformin by mouth are the immediate-release medicines or modified-release medicines [21]. At the start, the dosage for immediate-release metformin is $500 \mathrm{mg}$ two times per day orally or $850 \mathrm{mg}$ once per day orally, the maintenance dosage is $2000 \mathrm{mg}$ per day in divided doses and the maximum dosage is $2550 \mathrm{mg}$ per day [29]. For the extended-release, the initial dosage is 500 to $1000 \mathrm{mg}$ orally once a day and the maximum dosage is $2000 \mathrm{mg}$ [29]. Adult patients who have the standard-release metformin for up to $2 \mathrm{~g}$ per day, can start with the same daily dosage of metformin modified release, however, it is unsuitable if the dosage is more than $2 \mathrm{~g}$ per day for the standard-release tablets [23].

Metformin does not have a license to be used in children under 10 years [23]. For children (specialist use only) who are 10-17 years, initially a $500 \mathrm{mg}$ dosage of immediate-release medicines once per day is recommended by mouth. The modified-release medicine dose can be increased to $2 \mathrm{~g}$ per day by mouth, which is the maximum if needed [23]. For adults, initially $500 \mathrm{mg}$ once daily is recommended that can be increased if necessary up to $2 \mathrm{~g}$ once per day [23], except in Polycystic Ovary Syndrome (PCOS). Although metformin does not have a license for treating PCOS officially, it is used to treat the condition sometimes $[23,27]$. In PCOS, metformin can be given by mouth using immediate-release medicines then $1.5-1.7 \mathrm{~g}$ per day in two to three divided doses [23]. In premenopausal and anovulatory PCOS patients, metformin can cause ovulation. However, the maximum dosage for metformin immediate-release medicines in publications and the British National Formulary (BNF) differ from product license because differences can exist regarding the licenses of different formulations containing the same medicine [23].

\section{The Side Effects of Metformin}

Metformin has proven to be safe and effective and is usually well tolerated. However, many patients cannot tolerate the medication due to its side effects. Therefore, optimal metformin use requires a clear understanding of its side effects and safety [30]. Metformin oral tablets can cause side effects that can be mild or serious [23]. The usual side effects include nausea, abdominal bloating, flatulence, vomiting, diarrhoea/constipation, heartburn, headache, agitation, chills, dizziness, tiredness, abdominal cramps or pain, loss of appetite, asthenia, myalgia, upper respiratory tract infection and an altered or metallic taste [23,27]. However, evidence shows that the symptoms which are gastrointestinal (GI) $[11,31]$ and symptoms of the digestive tract [32] are mostly observed with metformin treatment. GI symptoms were confirmed to be more in participants who had metformin compared to the ones who had placebo (average $28 \%$ versus $16 \%$, $\mathrm{p}=0.01$ ) [33]. The reason for this could be because the microbiome within the intestine changes due to metformin [26]. One of the main sites of metformin action is the liver, however current research shows that metformin also affects the gut due to an association with the gut-brain-liver axis. The bile acids in the intestine increase with metformin, which can affect the microbiome and as a result, the secretion of GLP-1, cholesterol levels and stool consistency are affected [26].

One study reported that the GI side-effects were linked with metformin in approximately $25 \%$ of patients and about $5 \%$ of patients could not tolerate metformin [34]. In contrast, another study reported that although GI symptoms occurred in about $20-30 \%$ of patients with metformin, less than $5 \%$ of patients needed the medication to be stopped [4]. The GI side- effects are usually observed with metformin at the beginning and in some patients they can continue, especially those who are on very high doses [23]. However, GI symptoms have been found to decrease over time [35]. The uptake of metformin largely depends on the dosage and transporter [26]. Some studies argue the uptake of metformin and genetic inheritance and variation in the transporters can affect its tolerability [36]. Tolerance can be improved by increasing the dose slowly [23]. If GI side effects with standard initial drug treatment are experienced by an adult with T2DM, standard-release metformin should be offered to them as the initial drug treatment, with a steady increase of the dose over several weeks [7].

In contrast to the sulphonylureas, hypoglycaemia is not usually caused by metformin [6]. However, if metformin is used along with insulin or other anti-diabetic medications, low blood glucose levels can occur. Additionally, hypoglycemia can occur with increased exercise, alcohol consumption or not consuming enough food. Some of the symptoms of hypoglycemia include hunger, palpitations, trembling, anxiety, sweating, nervousness, irritability, moodiness, dizziness, headache and blurred vision. Metformin can impair calcium intake/absorption [35], cause weight loss or asthenia [37]. Metformin causes rare side effects, which include hepatitis, skin reactions such as rashes, itching or flushing, decreased vitamin B12 absorption or Lactic Acidosis (LA), when it should immediately be discontinued [23]. The use of metformin in the long-term had associations with vitamin B12 deficiency and anaemia, as reported by the Diabetes Prevention Program (DPP) as well as the DPP Outcomes Study (DPPOS) [35]. The risks involved in getting vitamin B12 deficiency due to metformin were assessed by the DPPOS. They found that the average haemoglobin and haematocrit levels were slightly less in those who had metformin treatment in comparison with patients who had placebo [35]. Around $30 \%$ of people taking metformin experience vitamin B-12 deficiency in the long term [38]. Therefore vitamin B12 serum concentrations need to be monitored intermittently with long-term 
therapy in metformin-treated patients. Metformin has been used for quite a number of years, particularly in overweight or obese subjects with T2DM. In this category of subjects, the levels of blood glucose can be hard to manage by using methods that are non-pharmacological. Metformin wasthe first antidiabetic drug that decreased complications that were related to diabetes and general mortality in T2DM patients who were overweight or obese [39]. The improvements in glucose control due to metformin are achieved without causing any weight gain because metformin is weight neutral or has a weight reducing effect [11]. A systematic review found that treatment with metformin causes weight loss [13]. However, how metformin is associated with weight is not very clear and therefore various reasons and explanations for the fluctuations in weight exist. One reason could be due to the side effects of the drug, common GI symptoms, a frequent upset stomach or diarrhoea, which can all affect food intake negatively. Another reason could be because severe discomfort can make people have reduced hunger and eat less, thus leading to consumption of fewer calories. However, the Federal Drug Administration (FDA) did not approve metformin for losing weight and it is only available by prescription.

\section{Cautions \& Contraindications}

Metformin has been associated with a rare, metabolic condition called Lactic Acidosis (LA), which is very dangerous, often fatal and life-threatening $[40,41]$. Symptoms of LA are not very specific and patients can have tiredness, lethargy, weakness, diarrhoea, vomiting, nausea, abdominal pain, anorexia, blue or cold skin, chills, muscle pain, hypotension, fast or difficult breathing, dizziness, hyperventilation, severe drowsiness, a metallic taste in the mouth or slow or irregular heartbeat [31]. Studies have reported that LA occurs because of substantial tissue hypoperfusion and hypoxia [42,43]. In LA, blood lactate concentrations increase (over $45 \mathrm{mg} / \mathrm{dl}$ or $5.0 \mathrm{mEq} / \mathrm{L}$ ), the $\mathrm{pH}$ of blood decreases (below 7.35) and disturbances of the electrolytes occur with increased gaps in the anions [13].

The disease process of metformin-associated LA is not fully understood. Evidence suggests that metformin prevents the clearance of lactate in the liver by stopping the mitochondrial respiratory chain's complex I [44]. With increased levels of metformin, oxidative phosphorylation decreases and aerobic metabolism gets converted into anaerobic metabolism [45]. The kidneys normally excrete metformin however metformin can accumulate if there is renal impairment. This leads to increases in metformin concentrations and may lead to LA [46]. Increased production of lactic acid may also be caused due to haemodynamic instability and/or tissue hypoxia that could be linked with a severe overdose of metformin or because of any underlying cardiovascular or respiratory conditions. LA can also be caused due to a lack of clearance of lactate rather than due to increases in its production. In acute metabolic acidosis (which includes LA and diabetic ketoacidosis), metformin is contra-indicated [23].

In the 1970s in the United States, an earlier biguanide known as phenformin, was taken off the formulary as it showed risks that were associated with LA [47]. Approximately 40-64 cases of LA were reported per 100,000 patient-years and were found to have been associated with phenformin $[4,48]$. However, in contrast to phenformin, increased oxidation of glucose was observed with metformin without causing any significant alterations in the production of fasting lactate in peripheral tissues [49]. Moreover, another study reported that metformin is different compared to phenformin regarding its molecular structure and in pharmacokinetics [50]. Furthermore, the incidence of LA was found to be very low in patients who take metformin, in contrast to phenformin [51]. Metformin was taken off from the US market due to concerns over LA but was reintroduced in 1995, after it was proven to be effective and safe in decreasing levels of glucose in the blood [30]. Reports of the true incidence of metformin-induced LA however are not consistent. However, in metformin users, the overall incidence of LA seems to differ across studies from about three per one hundred thousand person-years to ten per one hundred thousand person-years of exposure [41]. A two-year review of 11,800 participants who were taking metformin found that only two participants had developed LA (incidence: nine patients per one hundred thousand person-years) [13], while other studies concluded that metformin usage had no associations with LA [39,40,51]. A study conducted in Denmark found that patients who were acutely hospitalized with LA had a $\mathrm{pH}$ of less than 7.35 and lactate levels of $\geq 2.0 \mathrm{mmol} / 1$ and the odds ratio after adjustment was 0.79 , with a $95 \%$ confidence interval of $0.54-1.17$ [40]. Another study emphasized that LA usually occurs because of drug overdoses, dehydration, heart attack or stroke, heavy alcohol consumption, congestive heart failure, serious infections, recent surgery, X-rays or other mechanisms of scanning, that required an injectable iodinated contrast drug and in people who were more than 80 years of age [31]. Metformin has contraindications for various hypoxemic, chronic conditions that have associations with LA, such as renal [41,52], hepatic or pulmonary diseases and heart disease [13]. Impaired renal function can cause metformin to accumulate. A systematic review found that lactate levels did not increase much in patients who had CKD which was mild to moderate, with their estimated glomerular filtration rate (eGFR) being $30 \mathrm{ml} / \mathrm{min}$ per $1.73 \mathrm{~m}^{2}-60 \mathrm{ml} / \mathrm{min}$ per $1.73 \mathrm{~m}^{2}$ [41]. However, data regarding LA in CKD are limited. Moreover, there were no randomized controlled trials carried out for metformin usage in people with impaired kidney function [41]. Therefore, whether the risks of developing LA due to metformin treatment in patients with T2DM are increased in comparison with other glucose-lowering treatments, remain debatable. Others argue that most of the cases of LA are observed in patients who have serious conditions like renal failure, which can cause LA rather than metformin $[53,54]$. Adhering to safe prescribing practices can reduce the risks of LA and risks can also be decreased by taking consideration of the precautions and contraindications to avoid metformin or lactate accumulating in the body [6]. Patients and their carers should be informed of the risk of LA and immediate medical attention should be sought if symptoms suchas dyspnoea, abdominal pain, muscle cramps, hypothermia or asthenia occur [23]. An extreme lack of energy, muscle weakness, severe tiredness, slow heartbeat, yellow skin, fast/shallow breathing, a general feeling of discomfort, feeling cold, pins and needles, sore tongue, mouth ulcers and disturbed vision are some of the warning signs [26]. Patients who have chronic heart failure or when other drugs that can impair renal function are used simultaneously with metformin should have their treatments administered carefully. Treatment should be interrupted if patients get dehydrated and metformin should be avoided in conditions that can cause renal functions to worsen or cause tissue hypoxia [23]. In hepatic impairment along with occurrence of tissue hypoxia, metformin should be withdrawn completely [23].

In T2DM, Diabetic Nephropathy (DN) is a microvascular complication [12,55] and accumulation of metformin because of renal 
insufficiency has been concerning. Some patients who take metformin can also be at risk of getting kidney damage. However, metformin was found to improve albuminuria in patients who had DN in comparison with glibenclamide in one study [56]. Additionally, metformin was found to stop the loss of diabetes-induced podocytes by preventing oxidative injury in an animal study [57]. However, in patients who were having metformin, a recent study found that the risk of acute kidney injury did not increase by baseline eGFR in comparison with the patients who were not having metformin [58]. Regarding metformin usage, abnormal kidney function is one of the main contraindications. The FDA guidelines in early 2016 contraindicated metformin usage in men who had serum creatinine levels of $1.5 \mathrm{mg} / \mathrm{dl}$ $(133 \mu \mathrm{mol} / \mathrm{L})$ or above and in women who had serum creatinine levels of $1.4 \mathrm{mg} / \mathrm{dl}(124 \mu \mathrm{mol} / \mathrm{L})$ or above $[7,12]$. However others argued that serum creatinine on its own does not accurately measure renal function in patients who are elderly or in others who have a decrease in their muscle mass, and therefore eGFR needed to be determined [7]. Metformin usage should be stopped for an eGFR value of less than $60 \mathrm{ml} / \mathrm{min}$ [7]. Metformin should be prescribed with caution and the dose should be reviewed if kidney functions deteriorate all of a sudden and in those who at risk of an eGFR lower than $45 \mathrm{ml} / \mathrm{min} / 1.73 \mathrm{~m}^{2}$ [7]. Treatment with metformin must be discontinued if the eGFR is lower than $30 \mathrm{ml} / \mathrm{min} / 1.73 \mathrm{~m}^{2}$ [7,59]. In adults who have reduced kidney function, metformin must be avoided if the eGFR value falls to lower than $30 \mathrm{ml} / \mathrm{min} / 1.73 \mathrm{~m}^{2}$ [23]. For patients who have T2DM and are on metformin, an eGFR value of more than $30 \mathrm{ml} / \mathrm{min} / 1.73 \mathrm{~m}^{2}$ is indicated currently [23]. Metformin should not be continued in patients who need iodinated contrast material intravenously or those who need to have a surgical procedure until renal functions arestable, with normal serum creatinine levels and urine output and without any circulatory compromise or fluid overload [7]. In children whose kidney functions are impaired, metformin should be discontinued if the eGFR is found to be lower than $30 \mathrm{ml} / \mathrm{min} / 1.73 \mathrm{~m}^{2}$ [23]. However, the doses can be adjusted and in children with moderate impairment, it is advised that dose reductions should be considered. In adults with moderate impairment, the dose should be reduced [23]. Patient parameters should be monitored annually. Twice a year renal functions needs to be evaluated in patients who start to deteriorate or who have other risk factors regarding renal impairment [23].

In patients who have a diagnosis of Chronic Kidney Disease (CKD) as well as T2DM, care should be taken when prescribing metformin because it can decrease kidney function and therefore close monitoring of renal functions is essential [12]. A study conducted in Taiwan found that the eGFR decreased more in patients who had T2DM and were on metformin for at least 6 months in comparison with participants who stopped metformin usage for approximately one hundred days [12]. The retrospective cohort study found that metformin treatment in patients with T2DM significantly worsened renal functions in patients with T2DM and moderate CKD [12]. However, this study had some limitations because firstly, causation could not be concluded from the results because the researchers did not include randomization, as it was not a clinical trial. Secondly, all the confounding factors, for example, duration of T2DM or liver disease, were not included in this single-centered study. A previous study found that metformin usage in patients who had T2DM and advanced CKD had significant associations with enhanced risks of all-cause mortality in comparison with participants who had T2DM but did not use metformin [60]. This was concerning because reduced renal functions in patients who had moderate $\mathrm{CKD}$ and were also taking metformin could have increased risks of getting toxic reactions with metformin. However, the FDA revised their warnings in April 2016, regarding metformin treatment in patients who had impaired kidney function and re-defined the renal impairment measurements in accordance with eGFR [61]. The revised guidelines specified that only in patients who have severe CKD and whose eGFR is lower than $30 \mathrm{ml} / \mathrm{min} / 1.73 \mathrm{~m}^{2}$ metformin usage is completely contraindicated. Therefore, metformin treatment is allowed in patients with moderate CKD, who have an eGFR value of $30-59 \mathrm{ml} / \mathrm{min} / 1.73 \mathrm{~m}^{2}$ [61].

\section{Drug Interactions, Warnings and Precautions for Metformin}

Drug interactions involving metformin that are clinically significant are not very common [62]. However, a few medications can interfere with metformin's actions. The concentrations of metformin can increase if cimetidine, frusemide or nifedipine are also administered simultaneously [62]. Dosages should be adjusted if a patient is on frusemide, prednisolone, oestrogen, progesterone, testosterone, contraceptive pills or other diabetes medicines and blood glucose levels may need to be checked more often [26]. Some medications excreted by renal tubular secretion, such as morphine, quinine, ranitidine, digoxin, quinidine, amiloride, procainamide, triamterene, vancomycin and trimethoprim, may be competing against metformin for being eliminated [62]. Therefore, patients who take metformin simultaneously with these agents need close monitoring for any toxic reactions [63].

Some drugs such as contrast media (iodinated), ethanol and ioversol have been found to interact seriously with metformin, however all possible interactions have not been included in this review. Contraindications include chronic heart failure, Diabetic Ketoacidosis (DKA), hypersensitivity, metabolic acidosis with or without coma, myocardial infarction (MI) abnormal creatinine clearance due to shock, septicemia or severe renal disease. Although MI is an important event however, compared with sulfonylureas, it is observed less with metformin. Serious allergic reactions to metformin are not very common however some inactive ingredients present may cause allergic reactions [31]. It is also possible to have anaphylaxis, which is a serious allergic reaction to metformin [26].

Metformin can be used in pregnancy for both pre-existing and gestational diabetes [23]. During pregnancy, it is usually safe to take metformin either on its own or when combined with insulin. Studies have shown no or minor risks for the usage of metformin during pregnancy [23]. However, treatment with metformin should not be continued in women who have gestational diabetes, after giving birth [23]. In women who have pre-existing diabetes, metformin can be used during breast-feeding [23]. Although metformin passes into breast milk, the amount is very small. There is a lack of sufficient evidence on metformin with herbal supplements and remedies. However, this review does not cover an extensive list of side effects and others can occur. Additionally, people taking metformin may/may not experience any or all of the above side effects [64].

\section{Conclusion}

In conclusion, stabilizing the levels of blood glucose is the ultimate target for the treatment of diabetes, which can be achieved with the correct drugs either alone or combined, along with a healthy 
lifestyle. Metformin is the first line therapy for T2DM and is generally a safe and effective treatment. Metformin can help the body to avoid complications of high blood glucose, such as kidney damage, diabetic neuropathy or retinopathy. It correctly metabolizes food and restores the body's ability to respond to insulin. A possible increased risk of CVD mortality exists and although rare, the serious side effect of LA can occur because metformin can accumulate in the body. This review discusses the possible side effects of metformin but everyone taking metformin may or may not experience any or all of them. In patients who have T2DM, metformin can cause an adverse effect on renal function and moderate CKD can occur. Metformin needs to be used with caution in patients who have trauma, fever, congestive heart failure, surgery, renal or hepatic impairment or in the elderly and metformin treatment should be stopped before any type of surgery.

\section{Funding Information}

No grant was received from any funding agencies in any sector for this review.

\section{Conflict of Interest}

None

\section{References}

1. WHO (2019) World Health Organization.

2. Fowler MJ (2008) Microvascular and macrovascular complications of diabetes. Clin Diabetes 26: 77-82.

3. American Diabetes Association (2002) Implications of the United Kingdom Prospective Diabetes Study. Diabetes Care 25: 28-32.

4. DeFronzo RA (1999) Pharmacologic therapy for type 2 diabetes mellitus. Ann Intern Med 131: 281-303.

5. International Diabetes Federation (2017) IDF Diabetes Atlas ( $8^{\text {th }}$ Edn.).

6. Dunn CJ, Peters DH (1995) Metformin. A review of its pharmacological properties and therapeutic use in non-insulin-dependent diabetes mellitus. Drugs 49: 721-749.

7. National Institute for Health and Clinical Excellence (NICE) (2015) Type 2 diabetes in adults: Management (NG28).

8. Danaei G, Finucane MM, Lu Y, Singh GM, Cowan MJ, et al. (2011) National, regional, and global trends in fasting plasma glucose and diabetes prevalence since 1980: Systematic analysis of health examination surveys and epidemiological studies with 370 country-years and $2 \cdot 7$ million participants. Lancet 378: 31-40.

9. International Diabetes Federation (2015) IDF diabetes atlas, $6^{\text {th }}$ edition.

10. Fischer J, Ganellin CR, Ganesan A, Proudfoot J (2010) Standalone drugs. In: Ganellin. Analogue-based drug discovery. Weinheim: Wiley-VCH Verlag $\mathrm{GmbH} \& \mathrm{Co}$.

11. Holman R (2007) Metformin as first choice in oral diabetes treatment: the UKPDS experience. Journ Annu Diabetol Hotel Dieu 13-20.

12. Hsu WH, Hsiao PJ, Lin PC, Chen SC, Lee MY, et al. (2018) Effect of metformin on kidney function in patients with type 2 diabetes mellitus and moderate chronic kidney disease. Oncotarget 9: 5416-5423.

13. Salpeter SR, Greyber E, Pasternak GA, Salpeter EE (2010) Risk of fatal and nonfatal lactic acidosis with metformin use in type 2 diabetes mellitus. Cochrane Database Syst Rev 20: CD002967.

14. DeFronzo RA, Goodman AM (1995) Efficacy of metformin in patients with non- insulin-dependent diabetes mellitus. The Multicenter Metformin Study Group. N Engl J Med 333: 541-549.
15. Johansen K (1999) Efficacy of metformin in the treatment of NIDDM. Meta-analysis. Diabetes Care 22: 33-37.

16. Hashimoto H, Mizushima T, Ogura T, Kagawa T, Tomiyama K, et al. (2016) Study on AAV-mediated gene therapy for diabetes in humanized liver mouse to predict efficacy in humans. Biochem Biophys Res Communications 478: 1254-1260.

17. Chakraborty A, Chowdhury S, Bhattacharyya M (2011) Effect of metformin on oxidative stress, nitrosativestress and inflammatory biomarkers in type 2 diabetes patients. Diabetes Res Clin Pract 93: 56-62.

18. Zheng J, Woo SL, Hu X, Botchlett R, Chen L, et al. (2015) Metformin and metabolic diseases: A focus on hepatic aspects. Front Med 9: 173-186.

19. Bailey CJ, Turner RC (1996) Metformin. New England Journal of Medicine 334: 574-579.

20. Bosi E (2009) Metformin--the gold standard in type 2 diabetes: what does the evidence tell us? Diabetes Obes Metab 11: 3-8.

21. Diabetes Prevention Program Research Group (2002) Reduction in the incidence of type 2 diabetes with lifestyle intervention or metformin. $\mathrm{N}$ Engl J Med 346: 393-403.

22. Diabetes Prevention Program Research Group (2005) Role of insulin secretion and sensitivity in the evolution of type 2 diabetes in the Diabetes Prevention Program: Effects of lifestyle intervention and metformin. Diabetes 54: $2404-2414$

23. National Institute for Health and Clinical Excellence (NICE) (2019) Metformin Hydrochloride.

24. Lorenzati B, Zucco C, Miglietta S, Lamberti F, Bruno G (2010) Oral Hypoglycemic Drugs: Pathophysiological Basis of Their Mechanism of ActionOral Hypoglycemic Drugs: Pathophysiological Basis of Their Mechanism of Action. Pharmaceuticals (Basel) 3: 3005-3020.

25. Bristol NJ: Bristol-Myers Squibb (2009) Glucophage (metformin hydrochloride) and Glucophage XR (extended-release) prescribing information. Pg. no: $1-30$.

26. McCreight LJ, Bailey CJ, Pearson ER (2016) Metformin and the gastrointestinal tract. Diabetologia 59: 426-435.

\section{NHS UK (2019) Metformin.}

28. Metformin (Fortamet, Glucophage, Glucophage XR), Emedicine.com.

29. Drugs.com [Internet] (2019)

30. Flory J, Lipska K (2019) Metformin in 2019. JAMA 321: 1926-1927.

31. Nasri H, Rafieian-Kopaei M (2014) Metformin: Current knowledge. J Res Med Sci 19: 658-664.

32. Diabetes Prevention Program Research Group, Crandall J, Schade D, Ma Y, Fujimoto WY, et al. (2006) The influence of age on the effects of lifestyle modification and metformin in prevention of diabetes. J Gerontol A Biol Sci Med Sci 61: 1075-1081.

33. Diabetes Prevention Program Research Group (2012) Long-term safety, tolerability, and weight loss associated with metformin in the Diabetes Prevention Program Outcomes Study. Diabetes Care 35: 731-737.

34. Dujic T, Zhou K, Donnelly LA, Tavendale R, Palmer CN, et al. (2015) Association of organic cation transporter 1 with intolerance to Metformin in Type 2 diabetes: A GoDARTS study. Diabetes 64: 1786-1793.

35. Aroda VR, Edelstein SL, Goldberg RB, Knowler WC, Marcovina SM, et al. (2016) Long-term Metformin Use and Vitamin B12 Deficiency in the Diabetes Prevention Program Outcomes Study. J Clin Endocrinol Metab 101: 1754-1761.

36. Zhou K, Donnelly L, Yang J, Li M, Deshmukh H, et al. (2014) Heritability of variation in glycaemic response to metformin: a genome-wide complex trait analysis. Lancet Diabetes Endocrinol 2: 481-487. 
37. National Health Service (NHS) UK (2019) Metformin.

38. Bell DS (2010) Metformin-induced vitamin B12 deficiency presenting as a peripheral neuropathy. South Med J 103: 265-267.

39. U.K. Prospective Diabetes Study Group (1998) United Kingdom Prospective Diabetes Study 24: a 6-year, randomized, controlled trial comparing sulfonylurea, insulin, and metformin therapy in patients with newly diagnosed type 2 diabetes that could not be controlled with diet therapy. Ann Intern Med 128: 165-175.

40. Aharaz A, Pottegård A, Henriksen DP, Hallas J, Beck-Nielsen H, et al. (2018) Risk of lactic acidosis in type 2 diabetes patients using metformin: A case control study. PloS One 13: 0196122.

41. Inzucchi SE, Lipska KJ, Mayo H, Bailey CJ, McGuire DK (2014) Metformin in patients with type 2 diabetes and kidney disease: a systematic review. JAMA 312: 2668-2675.

42. Olivia PB (1970) Lactic acidosis. American Journal of Medicine 48: 209225 .

43. Kreisberg RA (1980) Lactate homerostasis and lactic acidosis. Ann Intern Med 92: 227-237.

44. Owen MR, Doran E, Halestrap AP (2000) Evidence that metformin exerts its anti-diabetic effects through inhibition of complex 1 of the mitochondrial respiratory chain. Biochem J 348: 607-614.

45. Bruijstens LA, van Luin M, Buscher-Jungerhans PM, Bosch FH (2008) Reality of severe metformin-induced lactic acidosis in the absence of chronic renal impairment. Neth J Med 66: 185-190

46. Lipska KJ, Bailey CJ, Inzucchi SE (2011) Use of metformin in the setting of mild-to- moderate renal insufficiency. Diabetes Care 34: 1431-1437.

47. Aguilar CA, Wong B, Gomez-Perez FJ, Rull JA (1992) Combination daytime chlorpropamide-metformin/bedtime insulin in the treatment of secondary failures in non insulin dependent diabetes. Rev Invest Clin 44 $71-76$

48. Stang MR, Wysowski DK, Butler-Jones D (1999) Incidence of lactic acidosis in metformin users. Diabetes Care 22: 925-927.

49. Cusi K, Consoli A, DeFronzo RA (1996) Metabolic effects of metformin on glucose and lactate metabolism in noninsulin-dependent diabetes mellitus. J Clin Endocrinol Metab 81: 4059-4067.

50. Sulkin TV, Bosman D, Krentz AJ (1997) Contraindications to metformin therapy in patients with NIDDM. Diabetes Care 20: 925-928.
51. Tahrani AA, Varughese GI, Scarpello JH, Hanna FW (2007) Metformin, heart failure, and lactic acidosis: is metformin absolutely contraindicated? BMJ 335: 508-512.

52. Lalau JD (2010) Lactic acidosis induced by metformin: incidence, management and prevention. Drug Saf 33: 727-740.

53. Brown JB, Pedula K, Barzilay J, Herson MK, Latare P (1998) Lactic acidosis rates in type 2 diabetes. Diabetes Care 21: 1659-1663.

54. Misbin RI, Green L, Stadel BV, Gueriguian JL, Gubbi A, et al. (1998) Lactic acidosis in patients with diabetes treated with metformin. N Engl J Med 338: 265-266.

55. Tang SCW, Chan GCW, Lai KN (2016) Recent advances in managing and understanding diabetic nephropathy. F1000Res 5.

56. Amador-Licona N, Guizar-Mendoza J, Vargas E, Sanchez-Camargo G, Zamora-Mata L (2000) The short-term effect of a switch from glibenclamide to metformin on blood pressure and microalbuminuria in patients with type 2 diabetes mellitus. Arch Med Res 31: 571-575.

57. Kim J, Shon E, Kim CS, Kim JS (2012) Renal podocyte injury in a rat model of type 2 diabetes is prevented by metformin. Exp Diabetes Res 210821 .

58. Bell S, Farran B, McGurnaghan S, McCrimmon RJ, Leese GP, et al. (2017) Risk of acute kidney injury and survival in patients treated with Metformin: an observational cohort study. BMC Nephrol 18: 163.

59. Pedrós C, Ávila M, Gómez-Lumbreras A, Manríquez M, Morros R, et al (2019) Lactic acidosis associated with metformin in patients with moderate to severe chronic kidney disease: study protocol for a multicenter population-based case-control study using health databases. BMC Nephrol 20: 193 .

60. Hung SC, Chang YK, Liu JS, Kuo KL, Chen YH, et al. (2015) Metformin use and mortality in patients with advanced chronic kidney disease: national, retrospective, observational, cohort study. Lancet Diabetes Endocrinol 3: 605-614.

61. US Food \& Drug Administration (FDA) (2016).

62. Rojas LB, Gomes MB (2013) Metformin: an old but still the best treatment for type 2 diabetes. Diabetol Metab Syndr 5: 6.

63. Glucophage ${ }^{\circledR}$ and Glucophage, XR product information, Bristol-Myers Squibb Company, USA.

64. Metformin-side-effects, Diabetes UK 


\section{II \\ Hetario}

Journal of Anesthesia \& Clinical Care

Journal of Addiction \& Addictive Disorders

Advances in Microbiology Research

Advances in Industrial Biotechnology

Journal of Agronomy \& Agricultural Science

Journal of AIDS Clinical Research \& STDs

Journal of Alcoholism, Drug Abuse \& Substance Dependence

Journal of Allergy Disorders \& Therapy

Journal of Alternative, Complementary \& Integrative Medicine

Journal of Alzheimer's \& Neurodegenerative Diseases

Journal of Angiology \& Vascular Surgery

Journal of Animal Research \& Veterinary Science

Archives of Zoological Studies

Archives of Urology

Journal of Atmospheric \& Earth-Sciences

Journal of Aquaculture \& Fisheries

Journal of Biotech Research \& Biochemistry

Journal of Brain \& Neuroscience Research

Journal of Cancer Biology \& Treatment

Journal of Cardiology: Study \& Research

Journal of Cell Biology \& Cell Metabolism

Journal of Clinical Dermatology \& Therapy

Journal of Clinical Immunology \& Immunotherapy

Journal of Clinical Studies \& Medical Case Reports

Journal of Community Medicine \& Public Health Care

Current Trends: Medical \& Biological Engineering

Journal of Cytology \& Tissue Biology

Journal of Dentistry: Oral Health \& Cosmesis

Journal of Diabetes \& Metabolic Disorders

Journal of Dairy Research \& Technology

Journal of Emergency Medicine Trauma \& Surgical Care

Journal of Environmental Science: Current Research

Journal of Food Science \& Nutrition

Journal of Forensic, Legal \& Investigative Sciences

Journal of Gastroenterology \& Hepatology Research

Journal of Gerontology \& Geriatric Medicine
Journal of Genetics \& Genomic Sciences

Journal of Hematology, Blood Transfusion \& Disorders

Journal of Human Endocrinology

Journal of Hospice \& Palliative Medical Care

Journal of Internal Medicine \& Primary Healthcare

Journal of Infectious \& Non Infectious Diseases

Journal of Light \& Laser: Current Trends

Journal of Modern Chemical Sciences

Journal of Medicine: Study \& Research

Journal of Nanotechnology: Nanomedicine \& Nanobiotechnology

Journal of Neonatology \& Clinical Pediatrics

Journal of Nephrology \& Renal Therapy

Journal of Non Invasive Vascular Investigation

Journal of Nuclear Medicine, Radiology \& Radiation Therapy

Journal of Obesity \& Weight Loss

Journal of Orthopedic Research \& Physiotherapy

Journal of Otolaryngology, Head \& Neck Surgery

Journal of Protein Research \& Bioinformatics

Journal of Pathology Clinical \& Medical Research

Journal of Pharmacology, Pharmaceutics \& Pharmacovigilance

Journal of Physical Medicine, Rehabilitation \& Disabilities

Journal of Plant Science: Current Research

Journal of Psychiatry, Depression \& Anxiety

Journal of Pulmonary Medicine \& Respiratory Research

Journal of Practical \& Professional Nursing

Journal of Reproductive Medicine, Gynaecology \& Obstetrics

Journal of Stem Cells Research, Development \& Therapy

Journal of Surgery: Current Trends \& Innovations

Journal of Toxicology: Current Research

Journal of Translational Science and Research

Trends in Anatomy \& Physiology

Journal of Vaccines Research \& Vaccination

Journal of Virology \& Antivirals

Archives of Surgery and Surgical Education

Sports Medicine and Injury Care Journal

International Journal of Case Reports and Therapeutic Studies

Journal of Ecology Research and Conservation Biology

Submit Your Manuscript: http://www.heraldopenaccess.us/Online-Submission.php 